\title{
Relaciones entre dosis de veneno de Crotalus durissus terrificus, síntomas y letalidad, en ratas de distintos sexos y edades
}

\author{
Zorzo, L.R.; Ruiz, R.M.; Acosta O.C. \\ Laboratorio de Fisicoquímica, Facultad de Ciencias Veterinarias, UNNE, Sargento Cabral 2139, \\ Corrientes (3400), Argentina. Tel/fax 03783-425753. E-mail: patcom@vet.unne.edu.ar.
}

\begin{abstract}
Resumen
Zorzo, L.R.; Ruiz, R.M.; Acosta, O.C.: Relaciones entre dosis de veneno de Crotalus durissus terrificus, síntomas y letalidad, en ratas de distintos sexos y edades. Rev. vet. 20: 2, 103-109, 2009. Existen factores intrínsecos y extrínsecos que intervienen en la susceptibilidad de un individuo ante una intoxicación. El objetivo del presente trabajo fue conocer la influencia de factores individuales de ratas tratadas con diferentes dosis de veneno entero de Crotalus durissus terrificus (Cdt) de Argentina, establecer relaciones entre síntomas, letalidad, sexo y edad de los animales tratados. Se trabajó con ratas Wistar adultos, juveniles, infantes y neonatos (machos y hembras), sometiéndolas a una intoxicación aguda con dosis de 500, 700 y 900 $\mathrm{ug} / \mathrm{kg}$ de veneno. Se formaron grupos de 10 animales según edad, sexo y dosis utilizada; el periodo de exposición fue de 5 horas, durante el cual se observaron y registraron los signos y síntomas neurológicos. Para evaluar la relación entre los síntomas y la letalidad, los animales se agruparon en 3 categorías según los síntomas presentados. También se analizó el tiempo sobrevida y el tiempo de inicio de síntomas. Los resultados obtenidos demuestran que el veneno de $\mathrm{C} d t$ causa alteraciones en la sinapsis neuromuscular, evidenciadas por la presencia de parestesia, paresia, parálisis posterior y total. Se observó una relación directa entre letalidad y dosis en infantes, juveniles y adultos; en neonatos no hubo diferencia entre las dosis, indicando una mayor susceptibilidad en dicho estadio. Los resultados del análisis de los grupos formados según síntomas presentes, evidenciaron una relación entre el tiempo de aparición de síntomas y la gravedad de la intoxicación, indicando que cuando más tardíos fueron los síntomas, aumentó la probabilidad de que el accidente sea leve. Inversamente, a medida que el tiempo disminuyó se constató mayor gravedad de los síntomas y aumento de letalidad. Los resultados evidencian una clara relación entre la gravedad de la intoxicación y el tipo y número de síntomas, así como el tiempo de aparición de los síntomas.
\end{abstract}

Palabras clave: Crotalus $s p$, veneno, rata, síntomas, mortalidad.

\begin{abstract}
Zorzo, L.R.; Ruiz, R.M.; Acosta, O.C.: Relationship among dose, signs, and mortality of Crotalus durissus terrificus venom in rats of different sex and age. Rev. vet. 20: 2, 103-109, 2009. There are intrinsic and extrinsic factors that are involved in an individual susceptibility to envenoming. The aim of this work was to know the influence of individual factors in rats treated with different doses of whole venom of Crotalus durissus terrificus (Cdt) of Argentina, and to establish a relationship between neurological signs, lethality, sex and age. Adult, young, infant, and neonatal Wistar rats, males and females, were submitted to 500, 700 and $900 \mathrm{ug} / \mathrm{kg}$ of whole venom. Lots of 10 animals each were grouped according to age, sex, and dose. Exposition time to venom was 5 hours, during which rodents were observed and neurological signs became evident. Animals were grouped in 3 categories according to signs, to evaluate a possible relationship with lethality. Survival time as well as onset of signs, were considered. Results indicate that $C d t$ venom causes disturbances in the neuromuscular synapse, as evidenced by the presence of paresthesia, paresis, and paralysis. There was a direct relationship between lethality, dose, and the age of groups. It was demonstrated that infants are more susceptible to the venom. Analysis of the groups formed according to signs showed a relationship between the onset and the severity of poisoning: a late manifestation may indicate a less severe case, whereas an early onset suggests a severe envenoming. Our results show a clear relationship between the severity of poisoning with the type and number of signs and the beginning of them.
\end{abstract}

Key words: Crotalus sp, venom, rat, signs, mortality. 


\section{INTRODUCCIÓN}

Existen variaciones en las reacciones de biotransformación de un xenobiótico que dependen de un gran número de factores exógenos y endógenos, dentro de estos últimos genéticos y fisiológicos. En investigación la comparación de datos se torna compleja debido a diferencias en la biotransformación de compuestos entre diferentes especies, entre individuos de una misma especie pero diferentes edades e incluso sexo. Por ello deben ser considerados todos los factores capaces de afectar la biotransformación del compuesto en estudio.

Algunos xenobióticos actúan sobre receptores celulares y tal acción depende de la especie, edad del individuo, sexo, tipo de tejido, equilibrio y estado hormonal y nutricional, entre otros. La tasa metabólica de un individuo está determinada primariamente por sus factores genéticos, por la evolución y adaptación de éstos y por factores ambientales; además, la expresión y actividad de las enzimas del individuo también están influenciadas por diversos factores (edad, sexo, alimentación, estado fisiológico y patológico, entre otros) $5,8,12,21$.

El proceso de biotransformación de xenobióticos es catalizado principalmente por el sistema de monooxigenasas dependiente del citocromo P450. Este sistema se encuentra presente en diferentes tejidos como el riñón, pulmón, piel, intestino, corteza adrenal, testículos, placenta, encéfalo y otros, pero es principal y particularmente activo en el hígado 7,8,11,27. Cada individuo puede responder de manera distinta a las intoxicaciones, se sabe que la transformación de etilenglicol en acido oxálico es mayor en el gato, que en la rata y en el cobayo, siguiendo este orden. En general los carnívoros conjugan los metabolitos con ácido glucorónico, los herbívoros con aminoácidos y los omnívoros con cualquiera de ellos. Se considera que los animales de experimentación más similares a la especie humana son monos, ratas y cerdos ${ }^{23}$.

Algunas variaciones están ligadas al sexo, por ejemplo las ratas machos son más susceptibles que las hembras al daño hepático por cloroformo, al igual que el paratión y la warfarina son menos tóxicas para los machos. El desarrollo de la capacidad metabolizadora con respecto a la edad es complejo y varía para cada sustrato y para cada especie y sexo ${ }^{21,23}$.

El veneno de la "víbora de cascabel" Crotalus durissus terrificus $(C d t)$ de Argentina es considerado principalmente neurotóxico porque actúa sobre la placa neuromuscular impidiendo la normal transmisión del impulso nervioso ${ }^{4,22,26}$. Para ese veneno, muchas accciones (edematizante, necrótica, proteolítica, neurotóxica) fueron muy bien estudiadas, sin embargo no se hallaron trabajos que mencionen diferencias en las variables individuales ante una intoxicación con el veneno entero de $C d t$. Estudios realizados con veneno de escorpión evidenciaron diferencias significativas en la intoxicación según la variable edad ${ }^{13}$.

$C d t$ representa un ejemplo de variación entre diferentes especies en cuanto a la composición de su veneno y la fisiopatología desarrollada en el individuo accidentado. Estas características son de relevante importancia por las diferencias en la implicancia terapéutica, lo que motiva el estudio específico de cada una de las especies dentro del mismo género ${ }^{25}$. Algunas variaciones en las reacciones de biotransformación, pueden estar asociadas a las propiedades de los sistemas enzimáticos actuantes, con amplias diferencias entre los distintos organismos.

Por todo ello, el objetivo del presente estudio fue conocer la influencia de factores individuales como sexo y edad, sobre la sintomatología y letalidad de ratas tratadas con diferentes dosis de veneno entero de la serpiente $C d t$ que habita la región nordeste de Argentina.

\section{MATERIAL Y MÉTODOS}

Se trabajó con ratas Wistar machos y hembras de los grupos etarios neonatos, infantes, juveniles y adultos, a los que se les indujo una intoxicación aguda con dosis de 500, 700 y $900 \mathrm{ug} / \mathrm{kg}$ de veneno entero de $C d t$ previo estudio exploratorio de dosis respuesta; para ello fueron seleccionadas las dosis mencionadas debido a que dosis menores no causaban síntomas clínicos visibles. El veneno fue obtenido por extracción manual, desecado y conservado a $-20^{\circ} \mathrm{C}$.

Para evaluar las variables edad y sexo se formaron tres grupos según dosis utilizadas, cada uno de estos se subdividió en cuatro grupos según edades (neonatos, infantes, juveniles y adultos) y cada grupo etario se dividió a su vez en 2 grupos según el sexo (machos y hembras), por lo tanto se formaron en total 24 grupos de 10 animales cada uno (numero total de animales: 240).

La inoculación del veneno se realizó vía e.v. en la vena caudal de la cola, previa dilución en solución buffer fosfato sódico (PBS) a pH 7,4. Los animales neonatos fueron inoculados vía i.p. Los controles (respetando los grupos erarios: 24 grupos de 5 animales c/u, número total: 120) fueron inoculados por las mismas vías con igual cantidad de PBS. El período de exposición fue de 5 horas.

Los animales fueron observados desde el momento de la inoculación durante todo el período de exposición para el registro de letalidad y de signos/síntomas neurológicos como: parestesia, (se evaluó mediante el aumento del aseo del miembro afectado y sacudida del mismo antes de la deambulación), paresia (observación durante el deambular del animal sobre una mesa, arrastrando el/los miembros), hiperreflexia, (exageración de los movimientos y reflejos al realizar un chasquido de dedos a $30 \mathrm{~cm}$ de distancia del animal), ptosis palpebral, (caída del parpado superior de uno u ambos ojos), convulsiones (movimientos bruscos involuntarios), parálisis posterior (miembros posteriores estirados hacia atrás e inmóviles) y parálisis total (miembros posteriores estirados hacia atrás y los miembros anteriores contraídos bajo del cuerpo, inmóviles).

Según los síntomas presentes en los animales tratados se asignaron 3 grupos para el análisis de datos: 
en el grupo 1 se incluyeron todos los animales que presentaron parálisis total acompañados de síntomas como: parestesia, paresia, hiperreflexia, ptosis palpebral, convulsiones $\mathrm{y} / \mathrm{o}$ parálisis posterior pero siempre parálisis total presente. En el grupo 2 todos los animales que presentaron dos o más de los síntomas mencionados anteriormente pero sin llegar a la parálisis total. El grupo 3 fue conformado por los animales que no presentaron síntomas y/o los que presentaron al menos uno de los primeros síntomas mencionados (parestesia, paresia, hiperreflexia y ptosis palpebral) sin llegar a parálisis.

Dichos grupos fueron formados para evaluar la relación entre la presentación de síntomas y la letalidad. Los animales que no murieron una vez cumplidas las 5 horas post-inoculación fueron anestesiados y sacrificados. Se analizó el tiempo promedio de sobrevida de todos los animales que murieron, dentro del período de estudio establecido, en los distintos grupos etarios, sexo y diferentes dosis utilizadas. El tiempo promedio de aparición de los síntomas fue relacionado con la letalidad, en cada sexo y grupo etario. Para el análisis de datos se utilizó estadística descriptiva y se aplicó el test de Chi cuadrado para variables no paramétricas.

\section{RESULTADOS}

Con la inoculación de dosis de $500 \mathrm{ug} / \mathrm{kg}$ se produjeron alteraciones clínicas con variaciones de intensidad acorde a la edad, las cuales se evidenciaron en la sensibilidad (parestesia), por la ausencia parcial de movimientos voluntarios (paresia), por exaltación en la respuesta de reflejos (hiperrreflexia) y por cambios en la posición del párpado superior (ptosis palpebral). No se registraron convulsiones. Con relación a la deambulación, las ratas revelaron incapacidad de movimientos de miembros posteriores en todas las edades (parálisis posterior), en tanto que parálisis total se observó solo en neonatos e infantes.

La letalidad afectó a los neonatos; con esta dosis el tiempo promedio de sobrevida fue de 93,33 minutos. En cambio, infantes, juveniles y adultos sobrevivieron a la intoxicación en nuestras condiciones de trabajo y por el tiempo oportunamente planeado. La letalidad fue de $30 \%$ en los neonatos mientras que estuvo ausente en los demás grupos etarios. Los resultados expresados en porcentajes según presencia o ausencia de síntomas se observan en Tabla 1.

En las ratas inoculadas con $700 \mathrm{ug} / \mathrm{kg}$ de veneno de C $d t$ (Tabla 2) se evaluaron los parámetros antemencionados, observándose que en la respuesta de parestesia, los neonatos tampoco manifestaron alteración, los infantes respondieron con mayor intensidad invirtiéndose el número de juveniles y adultos que presentaron parestesia. En la respuesta de paresia, en neonatos se presentó una pequeña disminución, todos los infantes respondieron, en tanto que los juveniles y adultos lo hicieron con menor intensidad.

La valoración de hiperreflexia demostró que respondieron de igual manera infantes y adultos, en tanto
Tabla 1. Síntomas correspondientes a la dosis de 500 $\mathrm{ug} / \mathrm{kg}$ (en \%).

\begin{tabular}{lcccc}
\hline \multirow{2}{*}{ síntomas } & \multicolumn{4}{c}{ grupo etario } \\
\cline { 2 - 5 } & neonatos & infantes & juveniles & adultos \\
\hline parestesia & - & 40 & 10 & 30 \\
paresia & 60 & 50 & 60 & 30 \\
hiperreflexia & 70 & 60 & 80 & 70 \\
ptosis palpebral & - & 10 & 70 & 30 \\
convulsiones & - & - & - & - \\
parálisis posterior & 70 & 40 & 60 & 30 \\
parálisis total & 70 & 10 & - & - \\
\hline
\end{tabular}

Tabla 2. Síntomas correspondientes a la dosis de 700 ug $/ \mathrm{kg}$ (en \%).

\begin{tabular}{lcccc}
\hline \multirow{2}{*}{ síntomas } & \multicolumn{4}{c}{ grupo etario } \\
\cline { 2 - 5 } & neonatos & infantes & juveniles & adultos \\
\hline parestesia & - & 60 & 30 & 10 \\
paresia & 70 & 100 & 40 & 20 \\
hiperreflexia & 30 & 60 & 40 & 60 \\
ptosis palpebral & 70 & 50 & 20 & 30 \\
convulsiones & - & - & - & - \\
parálisis posterior & 70 & 50 & 40 & 40 \\
parálisis total & 90 & 10 & 10 & 10 \\
\hline
\end{tabular}

Tabla 3. Síntomas correspondientes a la dosis de 900 ug $/ \mathrm{kg}$ (en \%).

\begin{tabular}{lcccc}
\hline \multirow{2}{*}{ síntomas } & \multicolumn{4}{c}{ grupo etario } \\
\cline { 2 - 5 } & neonatos & infantes & juveniles & adultos \\
\hline parestesia & - & 30 & 40 & 10 \\
paresia & 40 & 90 & 50 & 20 \\
hiperreflexia & 80 & 60 & 80 & 40 \\
ptosis palpebral & 30 & 20 & 20 & 40 \\
convulsiones & - & 10 & 10 & 10 \\
prálisis posterior & 60 & 60 & 70 & 90 \\
parálisis total & 80 & 30 & 10 & 40 \\
\hline
\end{tabular}

que los animales restantes lo hicieron en menor número. La ptosis palpebral se presentó en todas las edades, afectando a mayor número de animales neonatos e infantes. Ningún grupo etario padeció convulsiones con esta dosis. La parálisis de miembros posteriores se presentó en todos los animales superando el $50 \%$ en neonatos e infantes y menores porcentajes en juveniles $\mathrm{y}$ adultos.

La parálisis total fue muy elevada en neonatos, en tanto que pocos animales la manifestaron en el resto de los grupos etarios. Esta dosis no produjo letalidad en infantes, pero sí en neonatos $(50 \%)$, juveniles $(20 \%)$ y adultos (20\%). El tiempo promedio de sobrevida fue de 178,8 min en neonatos, $33 \mathrm{~min}$ en juveniles y $58 \mathrm{~min}$ en adultos.

Los neonatos inoculados con dosis de $900 \mathrm{ug} / \mathrm{kg}$ de veneno de $C d t$ (Tabla 3) no padecieron parestesia; en el resto de los animales dicho síntoma se registró en porcentajes variables entre las distintas edades. La hiperreflexia fue elevada en todas las edades, en iguales 
Tabla 4. Porcentaje de letalidad según dosis de veneno.

\begin{tabular}{lcccc}
\hline \multirow{2}{*}{ dosis } & \multicolumn{4}{c}{ grupo etario } \\
\cline { 2 - 5 } & neonatos & infantes & juveniles & adultos \\
\hline $500 \mathrm{ug} / \mathrm{kg}$ & 30 & 0 & 0 & 0 \\
$700 \mathrm{ug} / \mathrm{kg}$ & 50 & 0 & 20 & 20 \\
$900 \mathrm{ug} / \mathrm{kg}$ & 10 & 10 & 40 & 40 \\
\hline
\end{tabular}

Tabla 5. Tiempo de sobrevida (min) según dosis de veneno.

\begin{tabular}{lllll}
\hline \multirow{2}{*}{ dosis } & \multicolumn{5}{c}{ grupo etario } \\
\cline { 2 - 5 } & neonatos & infantes & juveniles & adultos \\
\hline $500 \mathrm{ug} / \mathrm{kg}$ & 93 & - & - & - \\
$700 \mathrm{ug} / \mathrm{kg}$ & 178 & - & 33 & 58 \\
$900 \mathrm{ug} / \mathrm{kg}$ & 135 & 289 & 179 & 152 \\
\hline
\end{tabular}

Tabla 6. Relación entre sexo y letalidad expresada en porcentaje según grupo etario.

\begin{tabular}{|c|c|c|}
\hline grupo etario & sexo & letalidad \\
\hline \multirow{2}{*}{ neonatos } & 우 & 46,67 \\
\hline & $\hat{0}$ & 13,33 \\
\hline \multirow{2}{*}{ infantes } & 우 & 6,67 \\
\hline & $\hat{\sigma}$ & $0 \%$ \\
\hline \multirow{2}{*}{ juveniles } & q & 26,67 \\
\hline & $\widehat{0}$ & 13,33 \\
\hline \multirow{2}{*}{ adultos } & q & 6,67 \\
\hline & $\sigma^{\pi}$ & 33,33 \\
\hline
\end{tabular}

porcentajes para neonatos y juveniles, seguida de los infantes, siendo los adultos los más resistentes. La ptosis palpebral se manifestó en porcentajes semejantes en todos los animales. Juveniles y adultos presentaron convulsiones, que se manifestaron por contracciones violentas e involuntarias (tónico-clónicas), los neonatos e infantes no presentaron este síntoma al igual que en las dosis previas estudiadas.

La parálisis posterior estuvo presente en todas las edades, en elevado número en animales adultos (90\%), en tanto que en la parálisis total la respuesta se invirtió respecto a la anterior, siendo más sensibles los neonatos con el $80 \%$ de individuos afectados. Esta dosis produjo baja letalidad en neonatos e infantes (10\%) y el tiempo promedio de sobrevida para tales grupos fue de $135 \mathrm{y}$ 298 min respectivamente. En juveniles y adultos la letalidad fue algo mayor ( $40 \%$ ), con un tiempo promedio de sobrevida de 179,33 y 152 min respectivamente.

Con respecto a la letalidad de ratas de diferentes grupos etarios tratadas con distintas dosis (Tabla 4), se pudo observar que con la dosis más alta todos los grupos fueron afectados (juveniles y adultos con mayor severidad). Para $700 \mathrm{ug} / \mathrm{kg}$ el único grupo no afectado fue el de infantes y para $500 \mathrm{ug} / \mathrm{kg}$ el único grupo afectado fue el de los neonatos.
Del total de ratas intoxicadas con las diferentes dosis de todos los grupos etarios (Tabla 5), las que murieron dentro del período de observación establecido presentaron tiempos promedios de sobrevida cuyas diferencias fueron no significativas.

La relación entre letalidad y sexo en los diferentes grupos etarios, sin discriminar dosis (Tabla 6), demostró que en ratas adultas los machos fueron más sensibles que las hembras, situación que se invirtió en neonatos, infantes y juveniles.

Del total de animales tratados (sin discriminar edad, sexo ni dosis), aquéllos que murieron por la intoxicación tuvieron un tiempo promedio de inicio de síntomas de 6,41 min, que fue menor al del resto de los animales intoxicados pero que no murieron, cuyo promedio fue de $26,5 \mathrm{~min}$. Se relacionó también la letalidad con el tiempo de inicio de síntomas según grupo etario, sin discriminar dosis y sexo (Figura 1), observándose que el tiempo en el cual se iniciaron los síntomas fue marcadamente menor en los animales que murieron con respecto a los que sobrevivieron en los distintos grupos etarios salvo en neonatos, donde la diferencia no fue tan marcada.

$\mathrm{Al}$ formar tres grupos según los síntomas presentes se pudo observar que en el grupo uno (incluyó todos aquellos que presentaran parálisis total), el tiempo promedio de inicio de síntomas fue el menor (12,54 min), en el segundo grupo (sin parálisis total pero con un número importante de síntomas presentes), el tiempo promedio de inicio de síntomas aumentó $(15,91 \mathrm{~min})$ y en el grupo tres (no presentaban síntomas o solo alguno de los primeros), el tiempo promedio de inicio de síntomas fue marcadamente más largo (68,88 $\mathrm{min})$, lo que demuestra una relación directa entre la categoría de síntomas presentes y el tiempo de inicio de los mismos.

En la asignación de los animales por edad en los tres grupos formados para el análisis de datos según sintomatología, se observó que en los adultos juveniles e infantes la mayoría de los individuos correspondieron al grupo 2 (sin parálisis total), mientras que en los neonatos el 83,33\% pertenecieron al grupo 1, el 16,66\% al grupo 2 y ningún animal al grupo 3 . El mayor número de animales tratados sin discriminar edad pertenecieron al grupo 2. Los porcentajes de animales para cada

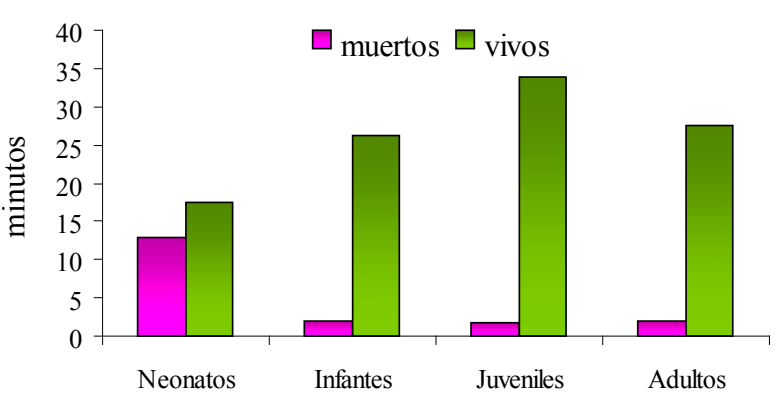

Figura 1. Tiempo de inicio $(\bar{x})$ de los síntomas relacionado con la letalidad registrada en cada grupo etario. 
grupo formado según grupo etario puede observarse en la Tabla 7.

Al aplicar el test de Chi cuadrado no se observaron diferencias significativas entre sexos pero sí entre grupos etarios, marcadamente en neonatos versus el resto de los grupos $(\mathrm{p}=0,00001)$.

\section{DISCUSIÓN}

La correspondencia entre la cantidad de un tóxico y la magnitud del efecto es lo que se conoce como la relación dosis-efecto o dosis-respuesta, siendo éste un concepto central de la toxicología. La magnitud y tipo de los efectos adversos producidos dependen de la dosis y duración de la exposición. En nuestro trabajo se planteó una duración de exposición de 5 horas debido a que las alteraciones neurológicas se instalan durante este período de tiempo ${ }^{3}$, siendo el objetivo observar sintomatología y letalidad en ese período con una exposición aguda al veneno en cuestión.

Existen diversos parámetros a tener en cuenta en la toxicidad que produce un compuesto, como ser su concentración en el sitio receptor, la biodisponibilidad determinada por propiedades físico-químicas del compuesto, la dosis y la metabolización. La concentración en el receptor depende de la especie animal, tipo de tejido, edad, sexo y estado hormonal ${ }^{17,21}$.

El veneno de $C d t$ se caracteriza por su neurotoxicidad periférica a nivel de la placa neuromuscular, también posee una enzima semejante a la trombina entre otros componentes, por lo que puede causar disturbios en la coagulación de la sangre ${ }^{16,24}$, incluyendo afibrinogenemia ${ }^{2}$.

En términos generales, los productos exógenos que afectan a la sinapsis se pueden clasificar en activadores (agonistas) o bloqueantes (antagonistas) de la neurotransmisión. El blanco de la crotoxina, principal componente neurotóxico del veneno de $C d t$, son los receptores colinérgicos nicotínicos que se encuentran en las placas motoras de los músculos voluntarios y los receptores neuronales del sistema nervioso central (SNC) y periférico. La crotoxina bloquea los receptores a partir de pocos minutos de su distribución en el organismo; la acción es progresiva provocando en algunos casos la muerte por paro respiratorio. Presumiblemente la toxina se metaboliza in situ, pues es sabido que una vez ejercido el efecto, la unión toxina-receptor no se rompe y si lo hace no mantiene su capacidad neutralizante de receptor, por tal razón las terminales neurológicas sufren un proceso degenerativo irreversible, que obliga a que el tratamiento se aplique dentro de las primeras 6 horas posteriores al accidente ${ }^{3}$.

Los resultados obtenidos en base a la sintomatología clínica demuestran que el veneno de $\mathrm{C} d t$ de Argentina causa alteraciones a nivel de la sinapsis neuromuscular produciendo una interrupción en la normal comunicación neuronal, evidenciada por la presencia de parestesia, paresia, parálisis posterior y total. Por otro lado cabe destacar la presencia de convulsiones
Tabla 7. Porcentaje de animales por grupo etario asignados a los grupos formados según síntomas presentes.

\begin{tabular}{lccc}
\hline \multirow{2}{*}{ grupo etario } & \multicolumn{3}{c}{ grupos formados según síntomas } \\
\cline { 2 - 4 } & 1 & 2 & 3 \\
\hline neonatos & 83,33 & 16,66 & 0 \\
infantes & 16,66 & 76,66 & 6,66 \\
juveniles & 6,66 & 73,33 & 20 \\
adultos & 16,66 & 50 & 33,33 \\
\hline
\end{tabular}

únicamente en animales intoxicados con la mayor dosis utilizada, estos resultados podrían ser atribuidos a la intoxicación del tronco cerebral a nivel de SNC, que en el ser humano cursa con mareos, visión borrosa, diplopía, ptosis palpebral, nistagmo, disminución de los reflejos fotomotores, oftalmoplejías y pérdida de relación con el medio, síntomas ligados a alteraciones presentes en los pares craneales III, IV, VI y VIII ${ }^{1,14}$.

Para confirmar esta hipótesis deberían realizarse estudios más específicos, ya que las convulsiones pueden tener origen craneal o extra craneal. Mecanismos secundarios provocados por el emponzoñamiento, como diversos grados de hemólisis, coagulación intravascular diseminada, mionecrosis, hemorragias, isquemia, aumento de la permeabilidad capilar y otros, pueden producir aumento de presión sanguínea en el SNC induciendo alteraciones en la liberación de neurotransmisores por activación de receptores centrales ${ }^{26}$.

En nuestro trabajo surge que existió relación entre la letalidad y las dosis utilizadas, es decir que a medida que aumenta la dosis también aumenta el porcentaje de animales muertos. Esta relación se cumple en los grupos de animales adultos, juveniles e infantes, mientras que en los neonatos la letalidad se produjo con todas las dosis utilizadas, demostrando una mayor sensibilidad al tóxico sin discriminación de dosis, datos que hasta el momento no habían sido confirmados con el veneno de cascabel.

Se ha demostrado que los neonatos y los animales muy jóvenes, en general son más susceptibles a los tóxicos, lo que se atribuye a deficiencias en varias enzimas de detoxificación, como sucede con el cloranfenicol, más tóxico para infantes por su deficiencia para formar glucorónidos ${ }^{23}$, también por la mayor absorción existente en la joven edad, así como al subdesarrollo de la barrera hematoencefálica y de los mecanismos de excreción, que producen acumulación de tóxicos. Sin embargo no todas las substancias son más tóxicas para esta edad; la disminución de la toxicidad también se debe a la ausencia de ciertas enzimas de bioactivación ${ }^{15}$. El efecto de la senectud sobre la toxicidad se ha estudiado poco, sin embargo se considera que se debe a la disminución de la capacidad de detoxificación y a la disminución de la excreción renal. La distribución de los tóxicos también puede cambiar por el incremento de la grasa y la pérdida de agua corporal ${ }^{23}$.

En nuestro trabajo, en el grupo de neonatos no se evidenció diferencia en los porcentajes de letalidad 
entre las distintas dosis ensayadas, resultados que estarían indicando una mayor susceptibilidad al veneno, ya que el número de muertos no varió con las dosis utilizadas. Muchos autores sugieren la existencia de diferencias en la actividad del citocromo P450 en función de la edad del individuo ${ }^{5,12}$, por ejemplo los animales jóvenes demostraron ser más sensibles a los efectos letales de plaguicidas organofosforados en comparación con los adultos, lo cual indica que los variados sistemas de detoxificación que existen en un organismo probablemente no respondan de igual manera según la edad del individuo ${ }^{6,10,15,18,19}$.

Por otro lado existe variabilidad en cuanto a las reacciones ante un tóxico según la variable sexo. Insecticidas organosfosforados como paratión demostraron ser más tóxicos en hembras que en machos. Ratas machos son más susceptibles al daño hepático provocado por halotano debido a la mayor velocidad con la que pueden transformar las sustancias en estructuras intermediarias reactivas. Ello también es válido para el riñón, donde los machos transforman el cloroformo diez veces más rápido que las hembras, causando mayor nefrotoxicidad en el macho.

Las concentraciones hormonales de machos y hembras influyen en la actividad del citocromo P450 7,9,11. Aparentemente los machos superan a las hembras en un 20 a $30 \%$ en la actividad y concentración de los sistemas enzimáticos de los microsomas hepáticos; algunas isoformas del citocromo P450 sólo se expresan en ratas machos, mientras que otras son específicas de las hembras ${ }^{20}$. En general las ratas machos metabolizan los xenobióticos mas rápidamente que las hembras, esto no ocurre en los ratones y podría interpretarse como diferencia ligada a la especie ${ }^{23}$.

En nuestros resultados la letalidad del grupo de los animales adultos fue mayor en los machos, sin embargo en el resto de los grupos etarios las hembras fueron las más sensibles, indicando que podría existir una combinación de factores como edad y sexo que podrían influir en estos resultados. Es importante recordar que el grupo de animales adultos se integró con adultos jóvenes (no gerontes), por lo que no deberían tener ningún trastorno en la detoxificación ligado a la edad adulta.

En seres humanos las diferencias del metabolismo de xenobióticos están menos influenciadas por el sexo que en algunas especies de animales de experimentación como las ratas, aunque hasta la fecha las isoformas sexo-específicas de los citocromos P450 no están bien identificadas en el hombre ${ }^{21}$.

Recordando que se conformaron tres grupos según síntomas presentes, en el grupo 3 el promedio de tiempo en el cual aparecieron dichos síntomas siempre fue mayor que en los grupos 1 y 2 . Es decir que a medida que se alarga el tiempo y no aparecen síntomas, hay más probabilidad de que el accidente no sea grave. Todo lo contrario sucedió cuando el tiempo promedio de aparición de síntomas se redujo, estableciéndose una relación directamente proporcional con la cantidad de muertes y la presencia de síntomas de mayor gravedad.
Esta relación se manifestó en animales adultos, juveniles e infantes. Sin tener en cuenta sexo, edad ni dosis, el menor tiempo de inicio de síntomas se relacionó al mayor porcentaje de animales muertos; ello ocurrió preponderantemente en los grupos 1 y 2 , claramente separados del grupo 3 quienes revelaron tardía aparición de síntomas y menores probabilidades de morir.

Debido a que entre los animales muertos ninguno perteneció al grupo 3, estos resultados podrían operar como indicativos de letalidad para evaluar la gravedad de un paciente emponzoñado, teniendo en cuenta que en un accidente ofídico no se conoce con exactitud la cantidad de veneno que la serpiente inocula en su víctima. No sucedió lo mismo con los neonatos ya que no se logró encontrar diferencia entre dosis utilizadas y promedio de tiempo de aparición de síntomas relacionados a la letalidad, indicando en todos los órdenes una mayor susceptibilidad en este grupo, independiente de dosis y tiempo en el que aparecen los síntomas, demostrado por la ausencia de individuos que pertenecieron al grupo 3.

Por lo tanto concluimos que el veneno de la especie $C d t$ de Argentina inoculado en ratas produce diferencias significativas en el grado de intoxicación según la edad, dato no corroborado hasta el momento en referencias bibliográficas. Por otro lado se pudo evidenciar una clara relación entre la gravedad de la intoxicación y el tipo de síntoma, así como el número y tiempo de aparición de síntomas en el animal intoxicado, datos de suma importancia para el diagnóstico clínico, interpretación fisiopatológica e implementación de un eficiente tratamiento.

\section{REFERENCIAS}

1. Acevedo Marquez MM, Cupo P, Amaral CF, Hering SE. 1990. Rattlesnake bites. Clinical feature and complementary test. Mem Inst Butantan 52, 27-30.

2. Amaral CF, da Silva OA, Lopez M, Pedroso ER. 1980. Afibrinogenemia following snake bite (Crotalus durissus terrificus). Am J Trop Med Hyg 29:1453-1455.

3. Barraviera B, Agapejev S, Castro CV. 1993. Importancia del diagnóstico clínico. Arg Bras Med 67: 36-37.

4. Bercovici D, Chudziniski AM, Dias W, Esteves MI, Hiraichi E, Oishi NY, Picarelli ZP, Rocha MC, Ueda CMPM, Yamanouye N, Row I. 1987. Asystematic fractionation of Crotalus durissus terrificus venom. Mem Inst Butantan 49: 69-78.

5. Blanco JG, Harrison PL, Evans WE, Relling MV. 2000. Human cytochrome $\mathrm{P}-450$ maximal activities in pediatric versus adult liver. Drug Metab Dispos 28: 379-382.

6. Brodeur J, DuBois KP. 1963. Comparison of acute toxicity of anticholinesterase insecticides to weanling and adult male rats. Proc Soc Exp Biol Med 114: 509-511.

7. Capdevila J, Harris RC, Falck JR. 2002. Microsomal cytochrome P450 and eicosanoid metabolism. Cell Mol Life Sci 59: 780-789. 
8. Coon MJ, Ding X, Pernecky SJ, Vaz AD. 1992. Cytochrome P450: progress and predictions. FASEB J 6: 669673.

9. Ding X, Kaminsky LS. 2003. Human extrahepatic cytochromes P450: function in xenobiotic metabolism and tissue-selective chemical toxicity in the respiratory and gastrointestinal tracts. Ann Rev Pharmacol Toxicol 43: 149-173.

10. Gaines TB, Linder RE. 1986. Acute toxicity of pesticides in adult and weanling rats. Fundam Appl Toxicol 7: 299308.

11. Goeptar AR, Sheerens H, Vermeulen NP. 1995. Oxygen and xenobiotic reductase activities of cytochrome P450. Crit Rev Toxicol 25: 25-65.

12. Gow PJ, Ghabrial H, Smallwood RA, Morgan DJ, Ching MS. 2001. Neonatal hepatic drug elimination. Pharmacol Toxicol 88: 3-15.

13. Januario MC, Campolina D. 1996. Acidentes por animals peçonhentos. In: Erazo Manual de Urgências em Pronto Socorro (Pires MT Ed.), Editora Médica e Científica, Rio de Janeiro, p. 740-752.

14. Lee CY, Lee SY. 1991. Cardiovascular effects of snake venoms. Snake Venom 52: 551-552.

15. Lu FC, Jessup DC, Lavallée A. 1965. Toxicity of pesticides in young versus adult rats. Food Cosmet Toxicol 3: 591-596.

16. Maruñak S, Acosta OC, Leiva L, Ruiz RM, Aguirre M, Teibler P. 2004. Mice plasma fibrinogen consumption by thrombin-like enzyme present in Crotalus durissus terrificus (rattlesnake) venom from the north-east region of Argentina. Medicina ( Buenos Aires) 64; 509-517.

17. Meibohm B, Beierle I, Derendorf H. 2002. How important are gender differences in pharmacokinetics? Clin Pharmacokinet 41: 329-342.

18. Moser VC, Padilla S. Hunter DL, Marshall RS, McDaniel KL, Phillips PM. 1998. Age- and gender-related differences in the time course of behavioral and biochemical effects produced by oral chlorpyrifos in rats. Toxicol Appl Pharmacol 149: 107-119.
19. Moser VC. 1999. Comparison of aldicarb and methamidophos neurotoxicity at different ages in the rat: behavioral and biochemical parameters. Toxicol Appl Pharm 157: 94-106.

20. Mugford CA, Kedderis GL. 1998. Sex-dependent metabolism of xenobiotics. Drug Metab Rev 30: 441-498.

21. Peña CE, Carter DE, Ayala-Fierro F. 2001. Toxicología ambiental: evaluación de riesgos y restauración ambiental. http://superfund.pharmacy.arizona.edu/toxamb/.

22. Prado-Franceschi J. 1990. On the pharmacology of covulcin and gyroxin. Mem Inst Butantan 52: 25-26.

23. Repetto Jiménez M. 1997. Toxicología Fundamental, $3^{\circ}$ ed., Diaz de Santos Ediciones, Madrid, p. 247-250.

24. Ruiz RM, Bongiovanni B, Leiva LC, Duffard AM, Rodríguez JP, Acosta OC, Duffard R. 2007. Neurotoxicological effects of a thrombin-like enzyme isolated from Crotalus durissus terrificus venom (preliminary study). Toxicon 50: 144-152.

25. Saravia P, Rojas E, Arce V, Guevara C, Lopez JC, Chaves E, Velásquez R, Rojas G, Gutierrez JM. 2002. Geographic and ontogenic variability in the venom of the neotropical rattlesnake Crotalus durissus: pathophysiological and therapeutic implications. Rev Biol Trop 50: 337-346.

26. Su MJ, Chang CC. 1984. Presynaptic effects of snake venom toxins which have phospholipase A2 activity (betabungarotoxin, taipoxin, crotoxin). Toxicon 22: 631-640.

27. Vences A, Labra N, Hernández N, Dorado V, Gómez J, Pérez I, Nosti R, Camacho R, Espinosa JJ. 2006. The effect of aspartame on rat brain xenobiotic-metabolizing enzymes. Hum Exp Toxicol 25: 453-459. 